\title{
Management of interstitial pregnancy in the era of laparoscopy: a meta-analysis of 855 case studies compared with traditional techniques
}

\author{
Greg Marchand, MD, FACS, FICS, FACOG ${ }^{1}$, Ahmed Taher Masoud, MD ${ }^{1,2}$, Anthony Galitsky, MD ${ }^{1}$, \\ Ali Azadi, MD, FACOG ${ }^{3}$, Kelly Ware, MS2 ${ }^{1}$, Janelle Vallejo, MS3 ${ }^{1}$, Sienna Anderson, BS ${ }^{1}$, Alexa King, BS ${ }^{1}$, \\ Stacy Ruther, BS 1 , Giovanna Brazil, BS ${ }^{1}$, Kaitlynne Cieminski, BS 1 , Sophia Hopewell, BS ${ }^{1}$, Kaitlyn Eberhardt, BS ${ }^{1}$, \\ Katelyn Sainz, MD ${ }^{1}$

\begin{abstract}
${ }^{1}$ The Marchand Institute for Minimally Invasive Surgery, Mesa, AZ, USA, ${ }^{2}$ Faculty of Medicine, Fayoum University, Fayoum, Egypt, ${ }^{3}$ Department of Urogynecology, Star Urogynecology, Peoria, AZ, USA
\end{abstract}

\begin{abstract}
Interstitial pregnancy is a rare, life-threatening condition that requires high clinical suspicion for diagnosis. Most cases are discovered after complications have occurred. Many authors have described laparoscopic management. Although previous systematic reviews have compared the attributes and complications associated with interstitial pregnancy, we endeavored to complete the first systematic review and meta-analysis to compare the laparoscopic treatment of interstitial pregnancy with the open approach in the modern age of laparoscopic surgery. We systematically searched PubMed, ClinicalTrials.gov, Scopus, Web of Science, and Cochrane until June 2020 using relevant keywords and screened them for eligibility. We found a statistically significant difference in blood loss between laparoscopic and open surgery (168 mL compared to 1,163 mL). Further, cumulative meta-analysis has revealed that blood loss in laparoscopy has been decreasing over time from 1991 to 2020. Laparoscopic patients took less operative time (63.2 minutes) compared to laparotomy patients (78.2 minutes). Patients in the laparoscopic group spent less time hospitalized (3.7 days) compared to laparotomy patients ( 5.2 days). Our findings add strength to the position that laparoscopic approaches to interstitial pregnancy can be considered first-line in most situations. The laparoscopic approach was found to have a mean blood loss of $168 \mathrm{~mL}$, and this blood loss seems to decrease over time. Increased gravidity and duration of amenorrhea are positive factors that increase bleeding during the procedure. We are unable to find enough high-quality data to significantly compare successful pregnancy following surgery or risk of mortality in these populations.
\end{abstract}

Keywords: Ectopic; Laparoscopy; Laparotomy; Meta-analysis

\section{Introduction}

Ectopic pregnancy is a pregnancy due to implantation outside the normal endometrium, and it is a rare occurrence representing about $2 \%$ of all pregnancies [1]. Many risk factors contribute to increasing the incidence of ectopic pregnancy, such as a history of ectopic gestation, tubal adhesions, history of endometriosis or pelvic inflammatory disease (PID), the use of some contraceptive devices, and previous tubal surgeries [2]. The most common sites within the fallopian tube include the ampulla (69.9\%), isthmus (12\%), and interstitium $(2.4 \%)[2,3]$. The term "interstitial pregnancy" is used interchangeably with "cornual pregnancy" to describe a pregnancy in the interstitium, although interstitial preg-

Received: 2020.09.29. Revised: 2020.12.01. Accepted: 2020.12.27. Corresponding author: Greg Marchand, MD, FACS, FICS, FACOG The Marchand Institute for Minimally Invasive Surgery, 10238 East Hampton, Street 212 Mesa, AZ 85209, USA

E-mail: www.marchandinstitute.org, gm@marchandinstitute.org https://orcid.org/0000-0003-4724-9148

Articles published in Obstet Gynecol Sci are open-access, distributed under the terms of the Creative Commons Attribution Non-Commercial License (http://creativecommons. org/licenses/by-nc/3.0/) which permits unrestricted non-commercial use, distribution, and reproduction in any medium, provided the original work is properly cited.

Copyright $\odot 2021$ Korean Society of Obstetrics and Gynecology 


\section{Obstetrics \& Gynecology Science}

Greg Marchand, et al. Systematic review of laparoscopy in interstitial pregnancy

nancy may be considered a more accurate description [4]. Some authors believe the term "cornual pregnancy" should be reserved to refer to gestations in a horn of a bicornuate uterus [5-7].

Symptoms of interstitial pregnancy vary from asymptomatic to non-specific symptoms of suprapubic pain, nausea, vomiting, and vaginal bleeding $[5,8]$. Therefore, diagnosing interstitial pregnancy presents a challenge for obstetricians; it requires high clinical suspicion, especially in women with the aforementioned risk factors. In the last two decades, there has been a noticeable rise in the incidence of interstitial pregnancies, mainly due to the increased use of contraceptive devices and increased prevalence of PID, in addition to the major advances in imaging technologies and ultrasound [9].

Treatment of cases of interstitial pregnancy range from medical treatment to surgical interventions. Local and systemic methotrexate is the mainline for medical treatment, mainly indicated in young nulliparous women desiring future fertility [9]. Surgical interventions include laparotomy and laparoscopic management.

Recently, laparoscopy has become more common for gynecologic and obstetric procedures as a safe approach with less bleeding and improved surgical outcomes $[10,11]$. A study by Gyr et al. [12] compared traditional abdominal hysterectomy with minimally invasive laparoscopic hysterectomy with an ultrasonic scalpel and found that the latter reduces the need for analgesia and improves postoperative outcomes. Laparoscopy has also shown considerable efficacy in the management of mild to moderate endometriosis [13] and is considered one of the safest gynecologic operations for removing ovarian masses in adolescents [14].

We aimed to conduct this systematic review and metaanalysis to provide an updated insight into the use of laparoscopy in interstitial pregnancy, in addition to measuring its effectiveness in reducing blood loss and other complications compared to traditional techniques. We endeavored to complete the first systematic review and meta-analysis to compare the laparoscopic treatment of interstitial pregnancy with the open approach in the modern age of laparoscopic surgery.

\section{Methods}

We conducted this systematic review and meta-analysis fol- lowing the guidelines reported in the Cochrane Handbook for Systematic Reviews of Interventions [15] and Preferred Reporting Items for Systematic Reviews and Meta-Analyses (PRISMA) statement [16].

\section{Literature search}

We searched for published studies in four electronic databases: PubMed, ClinicalTrials.gov, Web of Science, Scopus, and Cochrane Central Register of Controlled Trials (CENTRAL) up to June 2020. We used the following strategy for our search: (Laparoscop* OR cornuostomy OR cornu* OR laparotomy OR "cornual evacuation" OR "cornual resection" OR "cornual excision" OR "wedge resection" OR "loop ligature" OR "Vicryl loop placement" OR "conical exeresis" OR hysterectomy OR salping* OR traditional OR classic* OR conventional) AND ("interstitial pregnancy" OR "cornual pregnancy" OR "cornual gestation" OR "interstitial gestation" OR "cornual ectopic").

\section{Eligibility criteria}

All studies that met the following criteria were included: 1) patients=women with interstitial or cornual pregnancy; 2, 3) intervention with or without a comparator=all types of laparoscopic surgeries, open surgeries, or both, 4) outcomes=all reported outcomes, especially those regarding bleeding, operation time, and hospital stay; and 5) study design=all interventional and observational studies (cohort, case-control, cross-sectional, case series, and case reports). We excluded conference abstracts, non-English studies, reviews, and studies that report the effect of only one type of surgery. No restriction on age, place, or publication date was implemented.

\section{Screening and studies selection}

Retrieved citations were screened for eligibility in two steps: title and abstract screening in which preliminary eligible records from the first step entered the second one; followed by full-text screening in which the articles were assessed for all criteria to be included in our study. In addition, we manually screened the references of the included studies and previous 


\title{
Obstetrics \& Gynecology Science
}

\author{
Vol. 64, No. 2, 2021
}

systematic reviews for additional or missed citations.

\section{Data extraction}

After the screening step, we extracted the following data from the eligible studies using a formatted data extraction sheet: 1) summary and baseline characteristics of the patients in each study, including study design, type of operation, number of patients, groups, age, parity, gravidity, previous ectopic pregnancy, and duration of amenorrhea, and 2) any repeated outcomes (reported by two or more studies) including postoperative hospital stay (days), operation time (minutes), blood loss (mL), postoperative pregnancy rate, and ruptured ectopic pregnancy. Data for continuous outcomes were extracted as mean and standard deviation, and dichotomous outcomes were extracted as event and total.

\section{Quality assessment}

The quality of the included studies was assessed using four tools according to the study design. We assessed the quality of cohort, case series, and case control studies using three different tools developed by the National Heart, Lung, and Blood Institute [17]. Each tool consisted of questions to assess the risk of bias and confounders. These questions were answered by "yes," "no," "not applicable," "cannot determine," or "not reported," and then each study was given a score to guide the overall rating of the quality as "good," "fair," or "poor."

We also assessed the quality of the included case reports using the Joanna Briggs Institute (JBI) critical appraisal tool for case reports. The tool is composed of some questions developed according to the CARE Guidelines for reporting case reports. These questions were answered by "yes," "no," "not applicable," or "unclear" [18]. We could not assess publication bias due to the small number of included studies according to Egger's funnel-plot-based methodologies [19].

\section{Data synthesis}

Comprehensive meta-analysis and open meta-analyst software were used to perform this meta-analysis. Continuous outcomes were pooled as the mean and standard deviation (SD). Whenever studies provided median and range, we used the methods described by Hozo et al. [20]. to transform these data to mean and SD. Dichotomous data were pooled as proportions. A random-effects model was used. We employed meta-regression models and leave-one-out metaanalysis whenever there was significant heterogeneity. In addition, a cumulative meta-analysis was used to reveal trends in the data over time.

\section{Results}

\section{Literature search}

We identified 96 records after searching PubMed, ClinicalTrials.gov, Web of Science, Scopus, and CENTRAL. The remaining records after removing duplicates were screened for eligibility. Two hundred studies were included in the fulltext screening. We finally included 96 studies. We did not find any missing papers after screening the references of the included trials and previous systematic reviews.

\section{Characteristics of the included studies}

Our review included 96 studies: 65 case reports, 23 cohort studies, 6 case series, and 2 casecontrol studies conducted between 1992 and 2020 [7-115]. The included studies enrolled 885 patients. A total of 723 patients underwent laparoscopy, while 132 underwent operative laparotomy for the management of ectopic pregnancy. The median age of the included participants ranged from 19 to 42 years. A summary of the included studies and their findings and baseline characteristics of the enrolled patients is shown in Table 1.

\section{Results of risk of bias assessment}

According to JBI critical appraisal tool for case reports, the quality of the included reports ranged from moderate to high. Most of the included case reports did not clearly describe patient demographics. However, most of them have clearly reported patients' history, clinical presentation, diagnostic method, intervention, post-intervention status, and side effects, and provided takeaway lessons for clinical practice.

According to different $\mathrm{NIH}$ quality assessment tools for each study design, all case-control studies were of poor quality, while four case series were of fair quality, and the remaining two studies were of poor quality. Regarding cohort stud- 


\section{Obstetrics \& Gynecology Science}

Greg Marchand, et al. Systematic review of laparoscopy in interstitial pregnancy

Table 1. Study characteristics

\begin{tabular}{|c|c|c|c|c|c|c|c|c|}
\hline Study & $\begin{array}{l}\text { Type of } \\
\text { operation }\end{array}$ & $\begin{array}{l}\text { Study } \\
\text { design }\end{array}$ & $\begin{array}{c}\text { Number } \\
\text { of } \\
\text { patients }\end{array}$ & Age (yr) & Gravidity & Parity & $\begin{array}{l}\text { Duration } \\
\text { of amen- } \\
\text { orrhea }\end{array}$ & $\begin{array}{l}\text { Previous } \\
\text { ectopics }\end{array}$ \\
\hline Api and Api [74] (2010) & Laparoscopy & Case report & 1 & 38 & 3 & $0+2$ & & 1 \\
\hline Attia et al. [75] (2005) & Laparotomy & Case report & 1 & 34 & & & 17 & \\
\hline Bremner et al. [76] (2000) & Laparoscopy & Case report & 1 & 36 & 4 & 1 & 8.5 & 2 \\
\hline Cai et al. [77] (2009) & Laparoscopy & Case report & 1 & 32 & 5 & $0+4$ & 9.5 & \\
\hline Casadio et al. [78] (2009) & Laparoscopy & Case report & 1 & 27 & 3 & 2 & & \\
\hline Chachan et al. [79] (2011) & Laparoscopy & Case report & 1 & 36 & 3 & 1 & & \\
\hline Chauhan et al. [80] (2006) & Laparotomy & Case report & 1 & 40 & 3 & & 12 & \\
\hline \multirow[t]{2}{*}{ Chen et al. [94] (2019) } & Laparoscopy & $\begin{array}{l}\text { Retrospective } \\
\text { (cohort/analysis) }\end{array}$ & 14 & $32.8 \pm 5.9$ & $0.7 \pm 0.7$ & & & \\
\hline & Laparoscopy & & 26 & $33.0 \pm 5.5$ & $1 \pm 0.9$ & & & \\
\hline Cheng et al. [81] (2009) & Laparoscopy & Case report & 1 & 22 & 2 & 0 & & \\
\hline \multirow[t]{2}{*}{ Chin et al. [82] (2004) } & Laparotomy & Case report & 1 & 29 & 2 & 0 & 12 & \\
\hline & Laparotomy & & 1 & 34 & 1 & 0 & 17 & \\
\hline Choi et al. [96] (2009) & Laparoscopy & $\begin{array}{l}\text { Retrospective } \\
\text { (cohort/analysis) }\end{array}$ & 8 & & & & 7.6 & \\
\hline Corić et al. [83] (2004) & Laparoscopy & Case report & 1 & 42 & 3 & 2 & 5 & \\
\hline Cucinella et al. [86] (2012) & Laparoscopy & $\begin{array}{l}\text { Retrospective } \\
\text { (cohort/analysis) }\end{array}$ & 5 & 32 & 1.8 & & 7.2 & 1 \\
\hline Dendas et al. [84] (2017) & Laparotomy & Case report & 1 & 35 & & & & \\
\hline \multirow[t]{3}{*}{ Di Tizio et al. [85] (2018) } & Laparoscopy & Case report & 1 & 26 & & & & \\
\hline & Laparoscopy & & 1 & 30 & & & & \\
\hline & Laparoscopy & & 1 & 38 & & & & \\
\hline Divry et al. [21] (2007) & Laparotomy & Case report & 1 & 32 & & & & \\
\hline Dumesic et al. [22] (2001) & Laparotomy & Case report & 1 & 37 & 3 & 1 & & \\
\hline Faioli et al. [112] (2016) & Laparoscopy & Case series & 3 & & & & & \\
\hline Gao et al. [88] (2019) & Laparoscopy & $\begin{array}{l}\text { Retrospective } \\
\text { (cohort/analysis) }\end{array}$ & 9 & 30.1 & & & 4.3 & \\
\hline Garretto et al. [23] (2015) & Laparoscopy & Case report & 1 & & & & & \\
\hline Garzon et al. [24] (2019) & Laparoscopy & Case report & 1 & 30 & 2 & 1 & 12 & \\
\hline Gezer and Mutlu [25] (2004) & Laparoscopy & Case report & 1 & 36 & 2 & 1 & 7 & \\
\hline Grant et al. [100] (2017) & Laparoscopy & $\begin{array}{l}\text { Retrospective } \\
\text { (cohort/analysis) }\end{array}$ & 44 & 32.6 (NR) & 3 & 4 & 6 & \\
\hline Grimbizis et al. [26] (2004) & Laparoscopy & Case report & 1 & 28 & 3 & 2 & 7 & \\
\hline Grobman and Milad [27] (1998) & Laparoscopy & Case report & 1 & 31 & 3 & 4 & 7 & \\
\hline \multirow[t]{2}{*}{ Ghazali et al. [106] (2018) } & Laparoscopy & $\begin{array}{l}\text { Retrospective } \\
\text { (cohort/analysis) }\end{array}$ & 7 & $29.3 \pm 5.9$ & $2.9 \pm 0.7$ & & $8.4 \pm 2.1$ & 3 \\
\hline & Laparotomy & & 7 & $31.4 \pm 7.3$ & $2.7 \pm 1.5$ & & $11.0 \pm 4.6$ & 1 \\
\hline Huang et al. [108] (2005) & Laparoscopy & Case series & 4 & & & & 9 & \\
\hline \multirow[t]{2}{*}{ Hwang et al. [113] (2011) } & Laparoscopy & Case control & 54 & $32.74 \pm 5.11$ & & $0.98 \pm 0.74$ & $7.7 \pm 1.5$ & 6 \\
\hline & Laparoscopy & & 34 & $31.12 \pm 5.99$ & & $0.82 \pm 0.90$ & $8 \pm 2$ & 8 \\
\hline
\end{tabular}




\section{Obstetrics \& Gynecology Science}

Vol. 64, No. 2, 2021

Table 1. Continued

\begin{tabular}{|c|c|c|c|c|c|c|c|c|}
\hline Study & $\begin{array}{c}\text { Type of } \\
\text { operation }\end{array}$ & $\begin{array}{l}\text { Study } \\
\text { design }\end{array}$ & $\begin{array}{c}\text { Number } \\
\text { of } \\
\text { patients }\end{array}$ & Age (yr) & Gravidity & Parity & $\begin{array}{c}\text { Duration } \\
\text { of amen- } \\
\text { orrhea }\end{array}$ & $\begin{array}{l}\text { Previous } \\
\text { ectopics }\end{array}$ \\
\hline \multirow[t]{2}{*}{ Kahramanoglu et al. [110] (2017) } & Laparotomy & Case series & 1 & 25 & 4 & 2 & & \\
\hline & Laparoscopy & & 1 & 28 & 1 & 0 & 7 & \\
\hline \multirow[t]{2}{*}{ Kalchman and Meltzer [28] (1966) } & Laparotomy & Case report & 1 & 28 & 1 & 0 & & 1 \\
\hline & Laparotomy & & 1 & 29 & & & 8 & 1 \\
\hline Kasum et al. [29] (1998) & Laparotomy & Case report & 1 & 38 & 5 & 0 & 14 & 2 \\
\hline \multirow[t]{2}{*}{ Kim et al. [114] (2015) } & Laparoscopy & Case control & 26 & 32 & 0 & & $6(3-8)$ & \\
\hline & Laparoscopy & & 80 & 31 & 0 & & $6(4-10)$ & \\
\hline Kim et al. [101] (2016) & Laparoscopy & $\begin{array}{l}\text { Retrospective } \\
\text { (cohortanalysis) }\end{array}$ & 13 & $31(25-33)$ & $1(1-6)$ & $0(0-1)$ & $7(5)$ & \\
\hline Ko et al. [30] (2007) & Laparoscopy & Case report & 1 & 32 & 3 & 0 & & \\
\hline Koukoura et al. [31] (2020) & Laparoscopy & Case report & 1 & 39 & 1 & 0 & 9 & \\
\hline Kumakiri et al. [32] (2005) & Laparoscopy & Case report & 1 & 38 & 2 & 0 & 7.4 & \\
\hline Lai et al. [33] (2016) & Laparotomy & Case report & 1 & 22 & 2 & 1 & 12 & \\
\hline \multirow[t]{2}{*}{ Lam et al. [34] (2004) } & Laparotomy & Case report & 1 & 32 & & & 12 & 1 \\
\hline & Laparotomy & & 1 & 32 & & & 9 & 2 \\
\hline Lam and Tulandi [8] (1999) & Laparoscopy & $\begin{array}{l}\text { Retrospective } \\
\text { (cohort/analysis) }\end{array}$ & 22 & & & & & \\
\hline \multirow[t]{2}{*}{ Lazard et al. [107] (2011) } & Laparoscopy & Case series & 1 & 41 & 4 & 3 & 7 & \\
\hline & Laparoscopy & & 1 & 32 & 6 & 3 & 8 & \\
\hline Lee et al. [35] (2011) & Laparoscopy & Case report & 1 & 28 & 3 & 1 & & \\
\hline Lialios et al. [36] (2008) & Laparoscopy & Case report & 1 & 29 & 3 & 2 & 6.8 & \\
\hline Liao and Ding [37] (2009) & Laparotomy & Case report & 1 & 29 & 6 & 0 & 13 & \\
\hline \multirow[t]{2}{*}{ MacRae et al. [89] (2009) } & Laparoscopy & $\begin{array}{l}\text { Prospective } \\
\text { cohort }\end{array}$ & 10 & $30(7.5)$ & & & & 1 \\
\hline & Laparotomy & & 1 & 24 & & & & \\
\hline \multirow[t]{2}{*}{ Maher and Grimwade [38] (1982) } & Laparotomy & Case report & 1 & 31 & 3 & 2 & 6 & \\
\hline & Laparotomy & & 1 & 36 & 5 & 2 & 13 & \\
\hline Marfori and Kotzen [39] (2018) & Laparoscopy & Case report & 1 & 33 & 3 & 1 & 7.8 & \\
\hline \multirow[t]{2}{*}{ Morita et al. [40] (1997) } & Laparoscopy & Case report & 1 & 39 & 4 & 2 & 6 & \\
\hline & Laparoscopy & & 1 & 29 & & 0 & 7 & \\
\hline Mavrelos et al. (1996) & Laparoscopy & $\begin{array}{l}\text { Retrospective } \\
\text { (cohort/analysis) }\end{array}$ & 12 & 37 & & & 15 & \\
\hline Moon et al. [103] (2000) & Laparoscopy & $\begin{array}{l}\text { Retrospective } \\
\text { (cohort/analysis) }\end{array}$ & 24 & $33.4 \pm 5.8$ & & & & \\
\hline Moon et al. [104] (2010) & Laparoscopy & $\begin{array}{l}\text { Retrospective } \\
\text { (cohort/analysis) }\end{array}$ & 20 & 28 (NR) & & & 6.7 & \\
\hline Nabeshima et al. [41] (2010) & Laparoscopy & Case report & 1 & 38 & 2 & & & \\
\hline Ng et al. [98] (2009) & Laparoscopy & $\begin{array}{l}\text { Retrospective } \\
\text { (cohort/analysis) }\end{array}$ & 53 & $19-40$ & & & & \\
\hline Nirgianakis et al. [105] (2017) & Laparoscopy & $\begin{array}{c}\text { Retrospective } \\
\text { (cohort/analysis) }\end{array}$ & 10 & $34.5 \pm 6.21$ & $2.6 \pm 1.58$ & $1.0 \pm 1.05$ & & 2 \\
\hline
\end{tabular}




\section{Obstetrics \& Gynecology Science}

Greg Marchand, et al. Systematic review of laparoscopy in interstitial pregnancy

Table 1. Continued

\begin{tabular}{|c|c|c|c|c|c|c|c|c|}
\hline Study & $\begin{array}{l}\text { Type of } \\
\text { operation }\end{array}$ & $\begin{array}{l}\text { Study } \\
\text { design }\end{array}$ & $\begin{array}{c}\text { Number } \\
\text { of } \\
\text { patients }\end{array}$ & Age (yr) & Gravidity & Parity & $\begin{array}{l}\text { Duration } \\
\text { of amen- } \\
\text { orrhea }\end{array}$ & $\begin{array}{l}\text { Previous } \\
\text { ectopics }\end{array}$ \\
\hline \multirow[t]{2}{*}{ Oelsner et al. [42] (1993) } & Laparoscopy & Case report & 1 & 29 & & & & \\
\hline & Laparoscopy & & 1 & 21 & & & & \\
\hline \multirow[t]{3}{*}{ Olagundoye et al. [97] (2000) } & Laparotomy & $\begin{array}{l}\text { Retrospective } \\
\text { (cohort/analysis) }\end{array}$ & 7 & & & & & \\
\hline & Laparoscopy & & 66 & & & & & \\
\hline & Both & & 33 & & & & & \\
\hline Oral et al. [43] (2014) & Laparoscopy & Case report & 1 & 27 & & & & \\
\hline Osuga et al. [44] (2001) & Laparoscopy & Case report & 3 & 32 & 3 & 0 & 7 & \\
\hline Pasic et al. [45] (2002) & Laparoscopy & Case report & 1 & 21 & 1 & 0 & 8 & \\
\hline Pedroso et al. [46] (2014) & Laparotomy & Case report & 1 & 19 & 1 & 0 & 15 & \\
\hline Pluchino et al. [47] (2009) & Laparoscopy & Case report & 1 & 34 & 1 & 0 & 7 & 1 \\
\hline Poujade et al. [48] (2009) & Laparoscopy & Case report & 1 & 32 & 5 & & 9 & \\
\hline Pramayadi et al. (2018) & Laparoscopy & Case report & 1 & 35 & 2 & 1 & & \\
\hline $\begin{array}{l}\text { Quinlan and Newcombe [54] } \\
(2007)\end{array}$ & Laparoscopy & Case report & 1 & 40 & & & 6 & \\
\hline Raheem and Afifi [53] (2008) & Laparoscopy & Case report & 1 & 35 & 1 & & 8 & \\
\hline $\begin{array}{l}\text { Rheinboldt and Ibrahim [52] } \\
\text { (2013) }\end{array}$ & Laparoscopy & Case report & 1 & 20 & 1 & 0 & & \\
\hline Ron-el et al. [50] (1988) & Laparotomy & Case report & 1 & 38 & & & 14 & 1 \\
\hline \multirow[t]{2}{*}{ Ross et al. [55] (2006) } & Laparoscopy & Case report & 1 & 27 & 3 & 1 & 11 & \\
\hline & Laparoscopy & & 1 & 30 & 2 & 1 & 6 & \\
\hline Sagiv et al. [51] (2001) & Laparoscopy & Case report & 1 & 21 & 3 & 1 & 8 & 1 \\
\hline \multirow[t]{2}{*}{ Sagiv et al. [93] (2013) } & Laparotomy & $\begin{array}{l}\text { Retrospective } \\
\text { (cohort/analysis) }\end{array}$ & 5 & $33(20-47)$ & $5(1-9)$ & $2(0-5)$ & $6-17$ & \\
\hline & Laparotomy & & 8 & & & & & \\
\hline Sahoo et al. [56] (2009) & Laparotomy & Case report & 1 & 28 & 7 & $5+1$ & 5 & \\
\hline \multirow[t]{4}{*}{ Said [111] (2016) } & Laparoscopy & Case series & 1 & 23 & 1 & & & \\
\hline & Laparoscopy & & 1 & 30 & 4 & $1+2$ & & 2 \\
\hline & Laparoscopy & & 1 & 25 & & & 10 & \\
\hline & Laparoscopy & & 1 & 26 & 1 & 0 & 7 & \\
\hline Sant and Andersen [57] (2012) & Laparotomy & Case report & 1 & 30 & 2 & 0 & 21 & \\
\hline Sarmini and Tate [58] (2005) & Laparoscopy & Case report & 1 & 22 & 2 & 0 & & \\
\hline Sherer et al. [59] (1995) & Laparoscopy & Case report & 1 & 32 & 2 & 1 & 7 & \\
\hline \multirow[t]{2}{*}{ Soriano et al. [6] (2008) } & Laparoscopy & $\begin{array}{l}\text { Retrospective } \\
\text { (cohort/analysis) }\end{array}$ & 11 & $34.3 \pm 5.8$ & $3.7 \pm 1.8$ & $1.5 \pm 1.4$ & $59.1 \pm 14.7$ & 0.5 \\
\hline & Laparotomy & & 11 & $35.2 \pm 4.3$ & $4.3 \pm 2.1$ & $1.7 \pm 1.3$ & $49.7 \pm 8.4$ & \\
\hline Takeda et al. [60] (2009) & Laparoscopy & Case report & 1 & 29 & 3 & 2 & 7 & \\
\hline \multirow[t]{3}{*}{ Tinelli et al. [61] (2010) } & Laparoscopy & Case report & 1 & 34 & & & & \\
\hline & Laparoscopy & & 1 & 37 & & 2 & 11 & \\
\hline & Laparoscopy & & 1 & 31 & & & 7 & \\
\hline
\end{tabular}




\section{Obstetrics \& Gynecology Science}

Vol. 64, No. 2, 2021

Table 1. Continued

\begin{tabular}{|c|c|c|c|c|c|c|c|c|}
\hline Study & $\begin{array}{c}\text { Type of } \\
\text { operation }\end{array}$ & $\begin{array}{l}\text { Study } \\
\text { design }\end{array}$ & $\begin{array}{c}\text { Number } \\
\text { of } \\
\text { patients }\end{array}$ & Age (yr) & Gravidity & Parity & $\begin{array}{c}\text { Duration } \\
\text { of amen- } \\
\text { orrhea }\end{array}$ & $\begin{array}{l}\text { Previous } \\
\text { ectopics }\end{array}$ \\
\hline \multirow[t]{3}{*}{ Tulandi and Al-Jaroudi [90] (2004) } & Laparotomy & $\begin{array}{l}\text { Retrospective } \\
\text { (cohort/analysis) }\end{array}$ & 32 & & & & & 13 \\
\hline & Laparotomy & & 13 & & & & & $7.3 \pm 0.4$ \\
\hline & Laparoscopy & & 11 & & & & & $5.4 \pm 1.0$ \\
\hline Ugwumadu et al. [62] (1997) & Laparotomy & Case report & 1 & & 1 & & 33 & \\
\hline Uludag et al. [92] (2018) & Laparoscopy & $\begin{array}{l}\text { Retrospective } \\
\text { (cohort/analysis) }\end{array}$ & 3 & 30.6 (NR) & 1 & & 6 & \\
\hline Vicino et al. [63] (2000) & Laparoscopy & Case report & 1 & 39 & 7 & 3 & & \\
\hline Vilos [64] (1995) & Laparoscopy & Case report & 1 & 31 & 4 & 2 & 7 & \\
\hline Vilos [65] (2001) & Laparoscopy & Case report & 1 & 31 & & & 8 & \\
\hline Walid et al. (2010) & Laparoscopy & Case report & 1 & 27 & & & & \\
\hline Wang et al. [99] (2014) & Laparoscopy & $\begin{array}{l}\text { Retrospective } \\
\text { (cohort/analysis) }\end{array}$ & 9 & 30.4 & & & 7.2 & \\
\hline Watanabe et al. [91] (2014) & Laparoscopy & $\begin{array}{l}\text { Prospective } \\
\text { cohort }\end{array}$ & 13 & $34.6 \pm 10.2$ & & & $7.6 \pm 1.3$ & \\
\hline $\begin{array}{l}\text { Weissman and Fishman [67] } \\
\text { (1992) }\end{array}$ & Laparotomy & Case report & 1 & 34 & 2 & 1 & 20 & \\
\hline Wood and Hurley [68] (1992) & Laparoscopy & Case report & 1 & 27 & & & 6 & \\
\hline Woodland et al. [69] (1996) & Laparoscopy & Case report & 1 & 23 & & & 8 & \\
\hline Xu et al. [95] (2018) & Laparoscopy & $\begin{array}{l}\text { Retrospective } \\
\text { (cohort/analysis) }\end{array}$ & 14 & 30.9 & 2.21 & 0.14 & $5 \pm 7.1$ & \\
\hline Yalçın et al. [70] (2015) & Laparoscopy & Case report & 1 & 36 & 5 & 3 & 6 & \\
\hline Yang and Song [71] (2018) & Laparoscopy & Case report & 1 & 41 & & & 7 & \\
\hline Yoong et al. [109] (2020) & Laparoscopy & Case series & 12 & $31(20-44)$ & & & & \\
\hline Zhang et al. [72] (2004) & Laparoscopy & Case report & 18 & & & & $5-12$ & \\
\hline Zhang et al. [73] (2013) & Laparoscopy & Case report & 2 & 30.43 (NR) & 4,2 & 1.1 & 8,12 & \\
\hline Zuo et al. [87] (2012) & Laparoscopy & $\begin{array}{l}\text { Retrospective } \\
\text { (cohort/analysis) }\end{array}$ & 17 & $\begin{array}{l}26.8(20- \\
35)\end{array}$ & & & $7.8 \pm 0.7$ & \\
\hline
\end{tabular}

Data are reported as median (range) or mean \pm standard deviation, number.

NR, not reported.

ies, 13 studies were of fair quality, and 10 were poor quality.

\section{Analysis of the outcomes}

\section{1) Blood loss}

\section{Laparoscopy group}

Eighty-three studies reported blood loss in the laparoscopy group. The overall mean blood loss was $168 \mathrm{~mL}$ (confidence interval $[\mathrm{Cl}][141.3,194.7], P<0.001)$ (Fig. 1A). There was significant heterogeneity among these studies $\left(I^{2}=98.3 \%\right.$,
$P<0.001)$; therefore, a meta-regression model was employed. Gravidity and the duration of amenorrhea explained most of the heterogeneity among the included studies $\left(R^{2}=44 \%\right.$ and $51 \%$, respectively) (Supplementary Fig. 1). In addition, the cumulative meta-analysis showed a reduction in blood loss over time from 2000 to 2019 (Supplementary Fig. 2).

\section{Laparotomy group}

Data reported from 27 studies revealed a mean blood loss of $1,163 \mathrm{~mL}(\mathrm{Cl}[894.974,1431.023], P<0.001)$ in the laparotomy group (Fig. 1B). There was no heterogeneity among 


\section{Obstetrics \& Gynecology Science}

Greg Marchand, et al. Systematic review of laparoscopy in interstitial pregnancy

the data obtained from these studies $\left(I^{2}=0 \%, P=0.8\right)$.

\section{2) Operative time}

\section{Laparoscopy group}

Data on operative time in the laparoscopy group were reported by 52 studies. The overall mean operative time was 63.2 minutes $(\mathrm{Cl}[53.5,72.8], P<0.001)$ (Fig. 2A). However, data from these studies showed significant heterogeneity $\left(I^{2}=98.3 \%, P<0.001\right)$. Interestingly, a meta-regression model that included "duration of amenorrhea" as a covariate ex- plained $81 \%$ of the between-studies variance $\left(R^{2}=81 \%\right)$ (Supplementary Fig. 3). Cumulative meta-analysis of operative time showed a trend increase in the cumulative mean operative time from 46.5 minutes in 1996 to 63.3 minutes in 2019 (Supplementary Fig. 4).

\section{Laparotomy group}

Three studies reported data on operative time in the laparotomy group. No significant heterogeneity $\left(l^{2}=0 \%, P=0.3\right)$ was found among these studies, and the overall mean operative time was 78.2 minutes $(\mathrm{Cl}[68.2,88.2], P<0.001)$ (Fig. 2B).
A

Studies

Chen 2019

Chen 2019

Choi 2009

Cucinella 2012

Faioli 2016

Gao 2019

Hamid 2018

Hwang 2011

Hwang 2011

Kim 2014

Kim 2014

Kim 2016

Moon 2000

Moon 2000

Moon 2000

Moon 2000

Tulandi 2004

Uludag 2018

Yoong 2019

Zhang 2013

Zuo 2012

Wang 2014

Case reports

Overall $(\mid \wedge 2=98.31 \%, P<0.001)$

\section{B}

Studies

Hamid 2018

Sagiv 2013

Tulandi 2004

Case reports

Overall $(\mid \wedge 2=0 \%, P=0.760)$
Estimate (95\%, C.I.)

$519.000(-5.346,1,043.246)$

$1,192.000$ (809.541, 1,574.459)

$50.000(34.755,65.245)$

$47.000(40.689,53.311)$

$31.000(17.421,44.579)$

$24.440(15.143,33.737)$

$771.000(270.962,1,271.038)$

$282.500(261.723,303.277)$

805.000 (593.473, 1,016.527)

$142.500(105.869,179.131)$

$575.000(454.807,695.193)$

$852.500(383.268,1,321.732)$

$1,100.000(133.626,2,066.374)$

$133.000(-18.632,284.632)$

$32.000(25.927,38.073)$

$40.000(20.763,59.237)$

$460.000(418.220,501.780)$

$50.000(47.737,52.263)$

$1,050.000(494.674,1,605.326)$

$800.000(661.410,938.590)$

$75.300(41.122,109.478)$

$115.000(82.987,147.013)$

$262.000(175.727,348.273)$

$168.008(141.308,194.708)$

Estimate (95\%, C.I.)

$1,200.000(410.311,1,989.689)$

$700.000(-1,053.045,2,453.045)$

$1,385.700(853.628,1,917.772)$

$1,080.000(736.975,1,424.625)$

$1,162.999(894,974,1,431.023)$
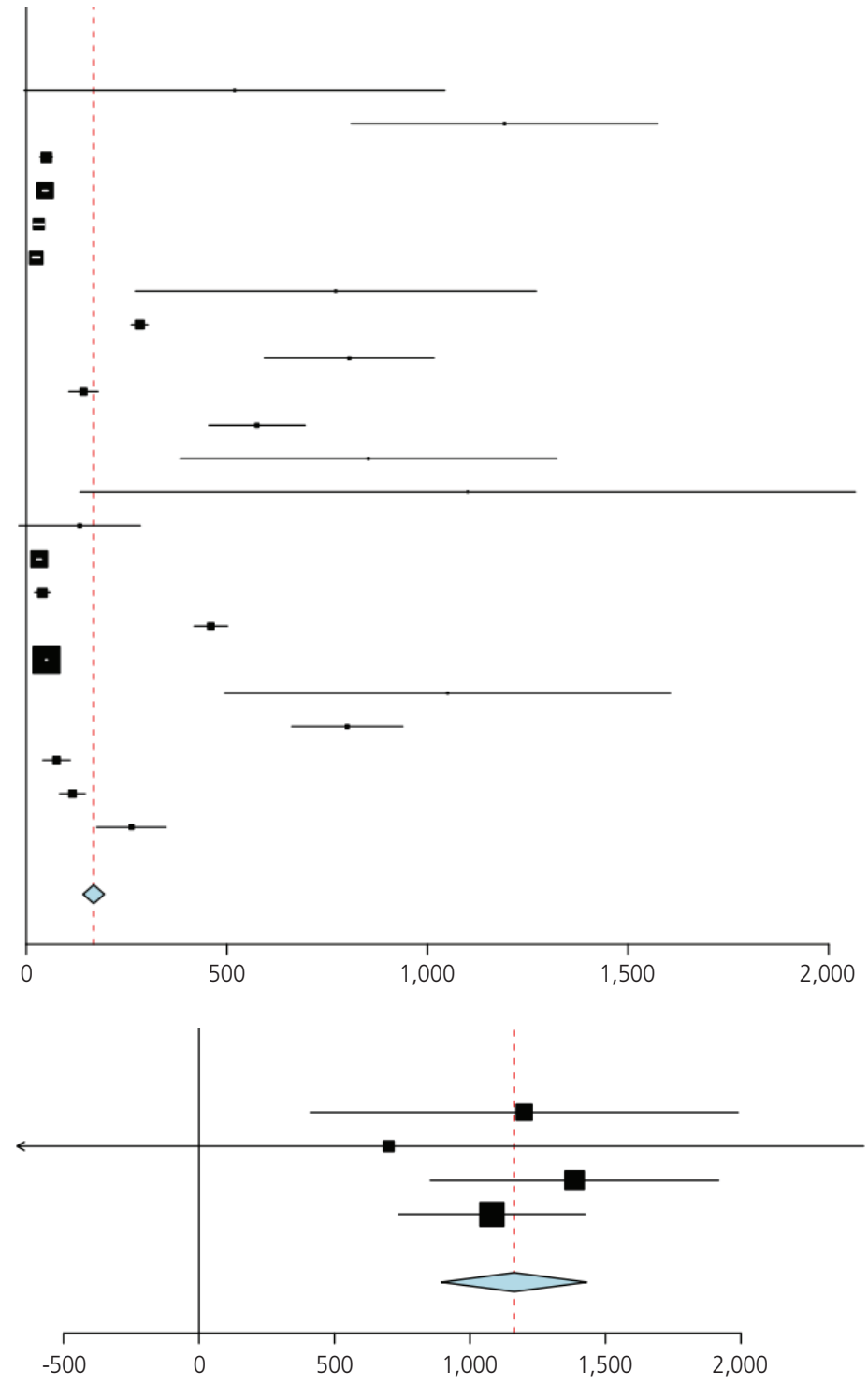

Fig. 1. Blood loss in the laparoscopy (A) and laparotomy (B) groups. 


\section{Obstetrics \& Gynecology Science}

Vol. 64, No. 2, 2021

\section{3) Hospital stay}

\section{Laparoscopy group}

Data on hospital stay in the laparoscopy group were reported by 48 studies. The overall mean hospital stay was 3.7 days (Cl $[2.7,4.7], P<0.001)$ (Fig. 3A). Considerable heterogeneity existed among the included studies $\left(I^{2}=98 \%, P<0.001\right)$. A meta-regression model that included "gravidity" and "year of the study" as covariates explained $95 \%$ of the between- studies heterogeneity $\left(R^{2}=0.95\right)$ (Supplementary Fig. 5).

\section{Laparotomy group}

Fifteen studies (12 case reports and 3 case series) were included in the meta-analysis for hospital stay in the laparotomy group (Supplementary Fig. 6). The summary estimate for mean hospital stay was 5.2 days $(\mathrm{Cl}[3,7.4], P<0.001)$ (Fig. $3 \mathrm{~B})$. There was significant heterogeneity among these studies $\left(I^{2}=96 \%, P<0.001\right)$. When the study by Ghazali et al. [106]
A

Studies

Estimate $(95 \%$, C.I.)

Chen 2019

Chen 2019

Choi 2009

Cucinella 2012

Faioli 2016

Gao 2019

Hamid 2018

Hwang 2011

Hwang 2011

Kim 2014

Kim 2014

Kim 2016

Mavrelos 1996

Moon 2000

Moon 2000

Moon 2000

Moon 2000

Moon 2010

$\mathrm{Ng} 2009$

OLAGUNDOYE 2000

Olagundoye 2000

Soriano 2008

Watanabe 2014

Xu 2018

Zhang 2013

Zuo 2012

Case reports

Overall $(\mid \wedge 2=98.28 \%, P<0.001)$

B

Studies

Hamid 2018

OLAGUNDOYE 2000

SORIANO 2008

Overall $(\mid \wedge 2=0 \%, P=0.285)$
$64.800(53.433,76.167)$

$83.800(72.768,94.832)$

$58.000(46.913,69.087)$

$39.000(35.231,42.769)$

$28.300(18.682,37.918)$

$70.440(55.819,85.061)$

$61.400(49.769,73.031)$

$71.300(62.338,80.262)$

$80.800(79.388,82.212)$

$62.500(53.659,71.341)$

$60.000(54.303,65.697)$

$78.750(62.279,95.221)$

$46.500(38.353,54.647)$

$82.500(24.110,140.890)$

$51.600(43.000,60.200)$

$35.000(29.342,40.658)$

$30.300(18.818,41.782)$

$120.000(104.466,135.534)$

$64.500(58.927,70.073)$

$58.200(51.990,64.410)$

$120.000(72.724,167.276)$

$107.000(94.497,119.503)$

$55.150(47.659,62.641)$

$95.000(32.634,157.366)$

$56.750(43.725,69.755)$

$61.300(49.615,72.985)$

$63.163(53.495,72.831)$

Estimate $(95 \%$, C.I.)

$97.100(68.802,125.398)$

$84.200(58.939,109.461)$

$73.600(61.781,85.419)$

$78.207(68.195,88.220)$
$28.500(25.261,31.739)$
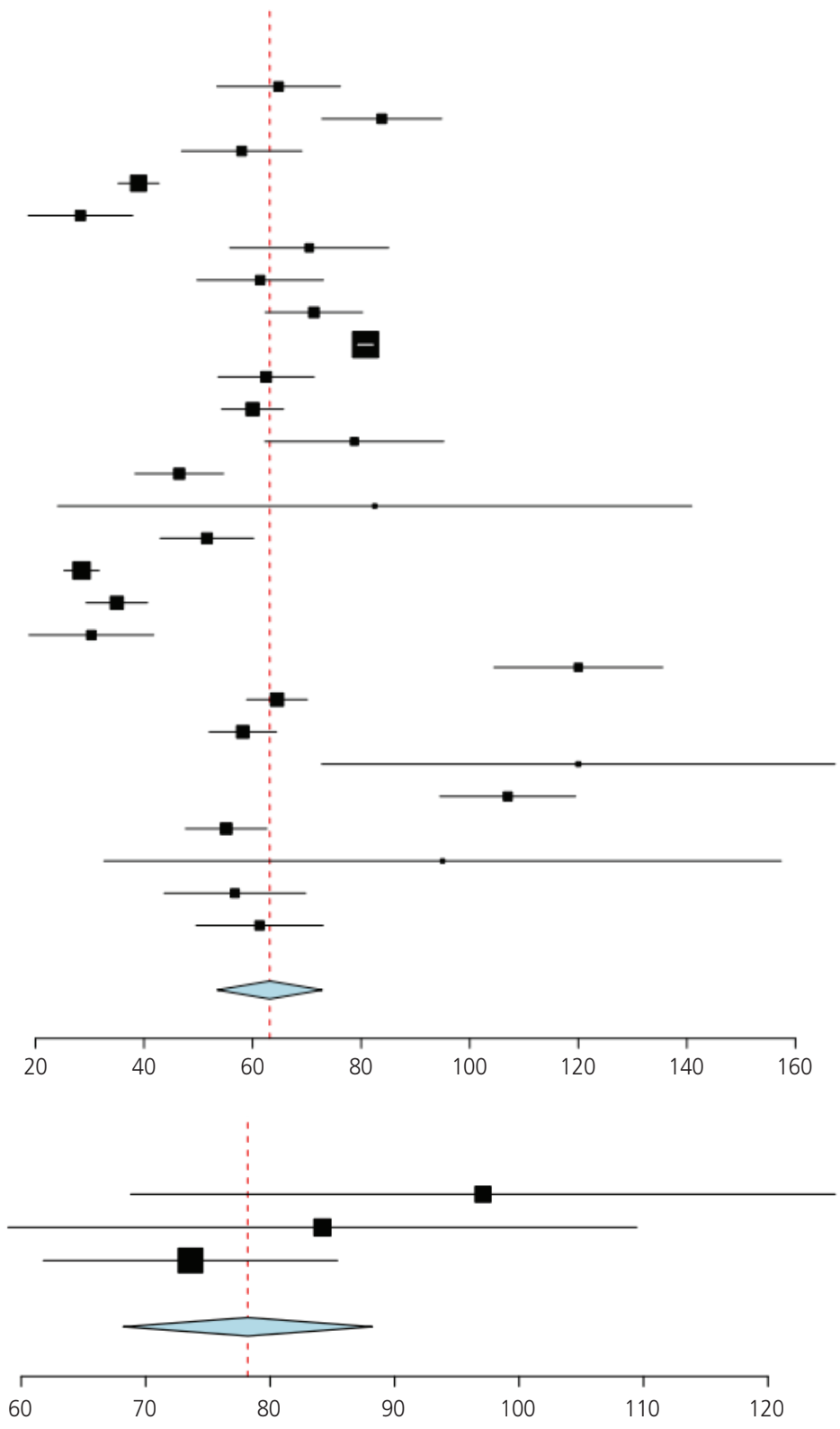

Fig. 2. Operative time in the laparoscopy (A) and laparotomy (B) groups. 


\section{Obstetrics \& Gynecology Science}

Greg Marchand, et al. Systematic review of laparoscopy in interstitial pregnancy

(2018) was removed in a leave-one-out meta-analysis, heterogeneity was minimal $\left(I^{2}=0 \%, P=0.5\right)$ (Supplementary Fig. 7).

\section{4) Incidence of rupture}

\section{Laparoscopy group}

Six studies provided analyzable data for the incidence of rupture in the laparoscopy group. The summary effect size was $0.51(\mathrm{Cl}[0.2,0.8], P<0.001)$ (Fig. 4A). There was significant heterogeneity among these studies $\left(I^{2}=91 \%, P<0.001\right)$. When the study of Watanabe et al. (2014) [91] was removed in a leave-one-out meta-analysis, heterogeneity was minimal $\left(I^{2}=0 \%, P=0.7\right)$ (Supplementary Fig. 8).

\section{Laparotomy group}

Only two studies that included 20 patients reported data on the incidence of ectopic rupture in the laparotomy group. The overall effect estimate was $0.77(\mathrm{Cl}[0.59,0.95]$, $P<0.001)$. No significant heterogeneity was found $\left(I^{2}=0 \%\right.$, $P=0.4$ ) (Fig. 4B).

\section{5) Future pregnancy rate}

\section{Laparoscopy group}

Data on future pregnancy rates were provided by 10 studies. The summary effect estimate was 0.62 (Cl $[0.42,0.82]$, $P<0.001)$. Significant heterogeneity was observed among these studies $\left(I^{2}=86.4 \%, P<0.001\right)$, and a meta-regression model was fitted to explain this heterogeneity. The duration of amenorrhea and sample size were responsible for almost all the heterogeneity among the effect estimates $\left(R^{2}=1\right)$.

\section{Laparotomy group}

The available studies provided no data on the future pregnancy rate in the laparotomy group.

\section{Discussion}

Our analysis showed less blood loss in the laparoscopic group $(168 \mathrm{~mL})$ than in laparotomy patients $(1,163 \mathrm{~mL})$. An
A

Studies

Hamid 2018

Hwang 2011

Hwang 2011

Kim 2014

Kim 2014

Kim 2016

Mavrelos 1996

Nirgianakis 2016

OLAGUNDOYE 2000

Soriano 2008

Case reports

Overall $(\mid \wedge 2=97.96 \%, P<0.001)$

$3.711(2.707,4.715)$

B

Studies

Hamid 2018

OLAGUNDOYE 2000

Soriano 2008

Case reports

Overall $(\mid \wedge 2=95.95 \%, P<0.001)$
Estimate $(95 \%$, C.I.)

$1.430(1.030,1.830)$

$5.800(5.320,6.280)$

$4.500(4.029,4.971)$

$4.400(3.631,5.169)$

$5.400(4.940,5.860)$

$4.300(3.485,5.115)$

$1.500(1.161,1.839)$

$2.670(2.050,3.290)$

$2.200(1.983,2.417)$

$5.700(3.513,7.887)$

$3.600(2.755,4.445)$

Estimate $(95 \%$, C.I.)

$2.570(1.985,3.155)$

$7.000(5.296,8.704)$

$5.900(5.309,6.491)$

$5.750(4.222,7.278)$

$5.238(3.037,7.440)$
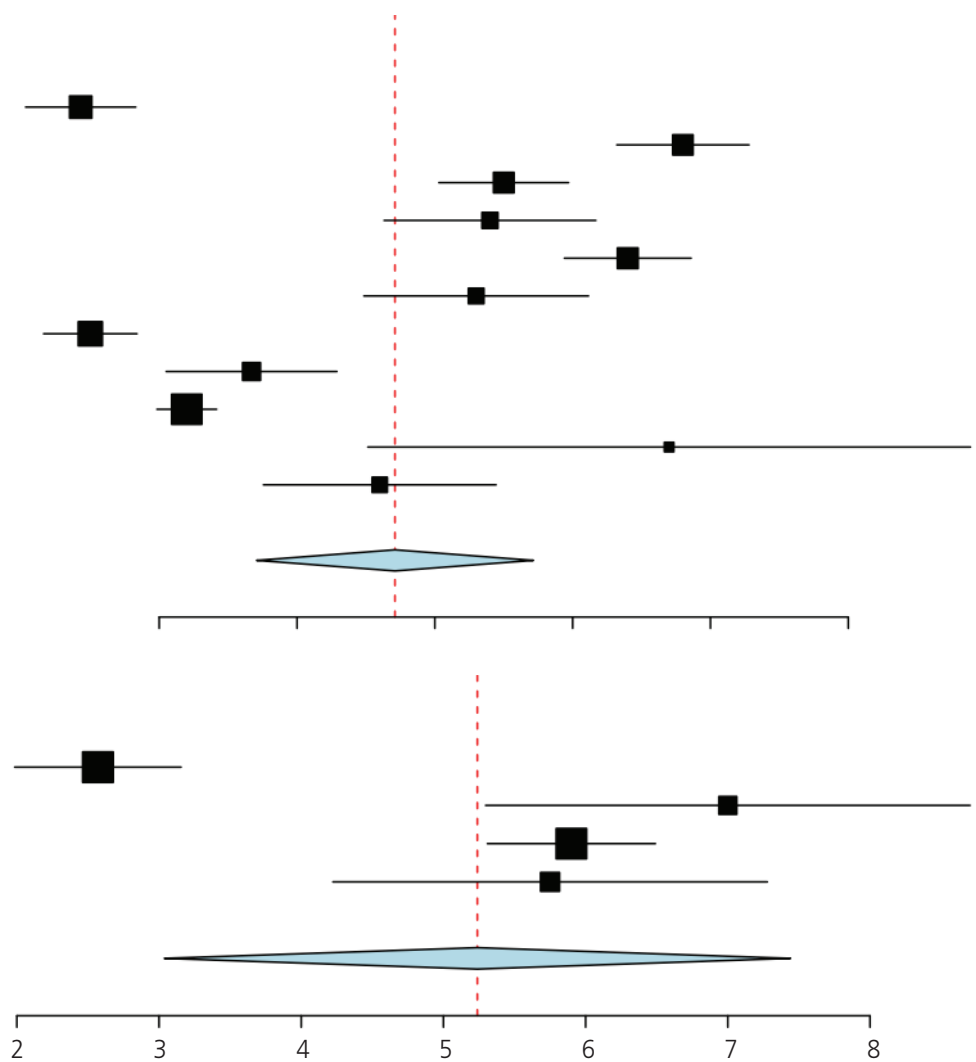

Fig. 3. Length of hospital stay in the laparoscopy (A) and laparotomy (B) groups. 


\title{
Obstetrics \& Gynecology Science
}

\author{
Vol. 64, No. 2, 2021
}

increased duration of amenorrhea and gravidity is associated with increased blood loss in laparoscopic patients. Interestingly, blood loss in laparoscopy has been decreasing over time, as shown by cumulative meta-analysis. This is consistent with the nature of laparoscopic operations. Laparoscopic patients took less operative time (63.2 minutes) compared to laparotomy patients (78.2 minutes). However, the mean operative time for the laparoscopic approach is actually increasing over time. Patients in the laparoscopic group spent less time hospitalized (3.7 days) compared to laparotomy patients who spent 5.2 days in the hospital on average. More recently performed studies and women with higher gravidity were both associated with a shorter hospital stay for patients in the laparoscopic group.

Laparotomy patients had a higher incidence of ectopic pregnancy rupture (77\%) than patients who underwent laparoscopic surgery (51\%). However, we could not employ a meta-regression model because of the low number of studies. The study by Watanabe et al. [91] (2014) significantly contributed to this heterogeneity as it reported a much lower incidence of ruptured ectopic pregnancy (0/13) than in other studies.

Only two previous systematic reviews have discussed the different management strategies, including laparoscopy and laparotomy $[103,104]$. These studies discussed and compared most medical and surgical options, but stopped short of a direct meta-analysis of the laparoscopic versus open approach. This is likely because these systematic reviews predate most of the high-quality data we were able to utilize to complete this analysis, being published in 2000 and 2010, respectively. Outside of isolating for interstitial pregnancy, many authors have completed reviews comparing open and laparoscopic ectopic pregnancies. For example, a meta-analysis by Gao et al. [116] compared laparoscopy and laparotomy for ectopic pregnancy and showed similar results regarding blood loss. However, they found no difference between laparoscopy and laparotomy in terms of operation time, which we found was lower in the laparoscopic group.

Both techniques have some advantages and drawbacks. Laparoscopy is a minimally invasive surgery and is associated with improved cosmesis, shorter hospital stay, faster recovery, less postoperative pain, and a higher rate of preservation of the uterus $[6,98,117]$. A laparoscopic approach, however, also has some drawbacks such as higher incidences of hematomas of the abdominal and abdominal or pelvic infections, although major complications are rarely reported [118]. There was not sufficient data to meaningfully analyze all possible laparoscopic techniques separately, and it is likely
A

Studies

Chen 2019

Chen 2019

Hamid 2018

Moon 2000

Tulandi 2004

Watanabe 2014

Overall $(\mid \wedge 2=91.45 \%, P<0.001)$

B

Studies

Hamid 2018

Tulandi 2004

Overall $(\mid \wedge 2=0 \%, P=0.370)$

$\begin{array}{ccc}\text { Estimate }(95 \%, \text { C.I. }) & \text { Ev/rrt } \\ & \\ 0.429(0.169,0.668) & 6 / 14 \\ 0.615(0.428,0.802) & 16 / 26 \\ 0.714(0.380,1.000) & 5 / 7 \\ 0.875(0.551,1.000) & 3 / 3 \\ 0.455(0.160,0.749) & 5 / 11 \\ 0.036(0.000,0.133) & 0 / 13 \\ & \\ 0.506(0.202,0.811) & 35 / 74\end{array}$

Estimate $(95 \%$, C.I.)

$0.857(0.598,1.000)$

$0.692(0.441,0.943)$

$0.772(0.592,0.952)$

Ev/Trt

$6 / 7$
$9 / 13$

$15 / 20$
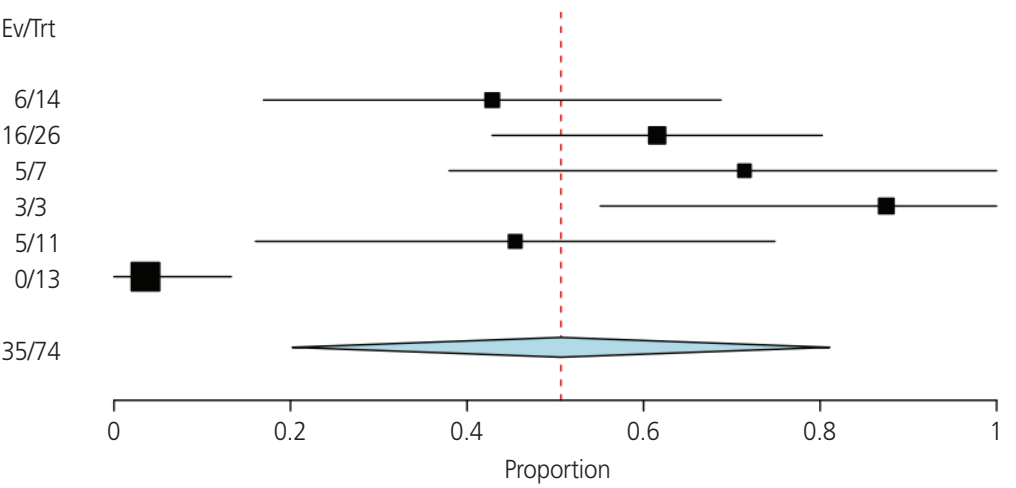

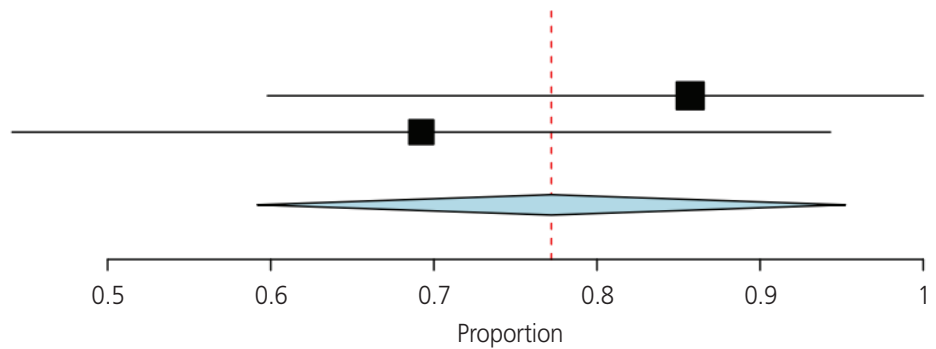

Fig. 4. Incidence of rupture in the laparoscopy (A) and laparotomy (B) groups. 


\section{Obstetrics \& Gynecology Science}

Greg Marchand, et al. Systematic review of laparoscopy in interstitial pregnancy

that the increased operative time for laparoscopic procedures from 1996 to 2019 was a result of more complex procedures and the advent of robotic-assisted laparoscopy.

Laparotomy is also a widely used management technique, especially when adequate closure or hemostasis cannot be achieved by laparoscopy, and when surgeons without laparoscopic expertise are available. Laparotomy, however, has multiple risks including the general risks of anesthesia and surgery, incisional hernia, serious infections, bleeding, and injury of pelvic or abdominal organs. All of these are reported more often in open approaches [106,113].

We included all studies reporting data regarding the safety and efficacy of laparoscopic surgery or laparotomy (or both) in the management of interstitial or cornual ectopic pregnancy patients, as indicated in our PRISMA flow chart (Supplementary Fig. 9). Additionally, most of the heterogeneity detected among the studies was managed. The quality of most of the included studies was fair, and a large number of studies entered the analysis, which increases the generalizability of the results. A cumulative meta-analysis was also used to reveal trends in the data over time.

Limitations of this study would include the lack of data regarding long-term effects, as we were able to find little or no data regarding late complications or overall survival. We reported what little we did find with regard to future pregnancy following surgical intervention. Another limitation is that all of the included studies were observational, which is generally considered at a low level of evidence. Lastly, the authors admit that the possibility of publication bias affecting results also exists.

\section{Conclusion}

Our analysis supports laparoscopy as the mainline surgical option for patients with interstitial pregnancy. Laparoscopy was associated with an average blood loss of $168 \mathrm{~mL}$, a mean operative time of one hour, and an average hospitalization time of 3.7 days. Our review shows that complications decrease over time. Interestingly, our analysis showed that both increased gravidity and duration of amenorrhea are positive risk factors leading to increased bleeding. Compared with laparotomy, management with laparoscopic surgery is associated with less blood loss, less operative time, and a shorter hospital stay. Laparotomy is also associated with a higher incidence of rupture of ectopic pregnancy.

Further interventional studies with a larger sample size and longer follow-up duration are needed to produce more valid results. We believe as the first systematic review to address this topic, our findings add strength to the position that laparoscopic approaches to interstitial pregnancy can be considered first-line in most situations.

\section{Supplementary materials}

Supplementary materials associated with this article can be found online at https://doi.org/10.5468/ogs.20299.

\section{Conflict of interest}

No potential conflict of interest relevant to this article was reported.

\section{Ethical approval}

The study was performed in accordance with the principles of the Declaration of Helsinki.

\section{Patient consent}

Written informed consent and the use of images from patients are not required for the publication.

\section{Funding information}

None.

\section{Acknowledgments}

The Marchand Institute for Minimally Invasive Surgery would like to acknowledge all the researchers, students, residents, and fellows who put time and effort without compensation into our projects for the benefit of women's health, and firmly assure them that the future of medicine belongs to them. 


\section{Obstetrics \& Gynecology Science}

Vol. 64, No. 2, 2021

\section{Condensation}

Many authors have described laparoscopic management of interstitial pregnancy. We performed the first systematic review of modern laparoscopy to assess the appropriateness of the laparoscopic approach as first-line management.

\section{IRB approval}

Found exempt at the Marchand Institute July 2020 IRB Meeting (2020-04-855).

\section{Prospero registration number}

CRD42020185248.

\section{References}

1. Jones T, Ho JR, Gualtieri M, Bruno-Gaston J, Chung K, Paulson RJ, et al. Clomiphene stair-step protocol for women with polycystic ovary syndrome. Obstet Gynecol 2018;131:91-5.

2. Attar E. Endocrinology of ectopic pregnancy. Obstet Gynecol Clin North Am 2004;31:779-94.

3. Stulberg DB, Cain LR, Dahlquist I, Lauderdale DS. Ectopic pregnancy rates and racial disparities in the Medicaid population, 2004-2008. Fertil Steril 2014;104:1671-6.

4. Ghaneie A, Grajo JR, Derr C, Kumm TR. Unusual ectopic pregnancies: sonographic findings and implications for management. J Ultrasound Med 2015;34:951-62.

5. Habana A, Dokras A, Giraldo JL, Jones EE. Cornual heterotopic pregnancy: contemporary management options. Am J Obstet Gynecol 2000;182:1264-70.

6. Soriano D, Vicus D, Mashiach R, Schiff E, Seidman D, Goldenberg M. Laparoscopic treatment of cornual pregnancy: a series of 20 consecutive cases. Fertil Steril 2008;90:839-43.

7. Faraj R, Steel M. Management of cornual (interstitial) pregnancy. Obstet Gynaecol 2007;9:249-55.

8. Lau S, Tulandi T. Conservative medical and surgical management of interstitial ectopic pregnancy. Fertil
Steril 1999;72:207-15.

9. Moawad NS, Mahajan ST, Moniz MH, Taylor SE, Hurd WW. Current diagnosis and treatment of interstitial pregnancy. Am J Obstet Gynecol 2010;202:15-29.

10. Levine DJ. Safety and efficacy of laparoscopy for benign gynecologic conditions. Am J Obstet Gynecol 2001;184:1585-6.

11. Tadir Y, Fisch B. Operative laparoscopy: a challenge for general gynecology? Am J Obstet Gynecol 1993;169:7-12.

12. Gyr T, Ghezzi F, Arslanagic S, Leidi L, Pastorelli G, Franchi M. Minimal invasive laparoscopic hysterectomy with ultrasonic scalpel. Am J Surg 2001;181:516-9.

13. Sulewski JM, Curcio FD, Bronitsky C, Stenger VG. The treatment of endometriosis at laparoscopy for infertility. Am J Obstet Gynecol 1980;138:128-32.

14. Grabowski A, Korlacki W, Pasierbek M. Laparoscopy in elective and emergency management of ovarian pathology in children and adolescents. Wideochir Inne Tech Maloinwazyjne 2014;9:164-9.

15. Higgins JP, Thomas J, Chandler J, Cumpston M, Li T, Page MJ, et al. Cochrane handbook for systematic reviews of interventions. 2nd ed. Chichester: John Wiley \& Sons, Ltd.; 2008.

16. Moher D, Liberati A, Tetzlaff J, Altman DG; PRISMA Group. Preferred reporting items for systematic reviews and meta-analyses: The PRISMA statement. PLoS Med 2009;6:e1000097.

17. National Institute of Health National Heart, Lung and Blood Institute. Quality Assessment Tools [Internet]. Bethesda (MD): National Institute of Health National Heart, Lung and Blood Institute; c2020 [cited 2020 Aug 2]. Available from: https://www.nhlbi.nih.gov/ health-topics/study-quality-assessment-tools.

18. Moola S, Munn Z, Tufanaru C, Aromataris E, Sears K, Sfetcu $R$, et al. Chapter 7: Systematic reviews of etiology and risk. [Internet]. Adelaide: JBI; C2020 [cited 2020 Aug 2]. Available from: https://wiki.jbi.global/ display/MANUAL/Chapter+7\%3A+Systematic+reviews +of+etiology+and+risk.

19. Egger M, Smith GD, Schneider M, Minder C. Bias in meta-analysis detected by a simple, graphical test. BMJ 1997;315:629-34.

20. Hozo SP, Djulbegovic B, Hozo I. Estimating the mean and variance from the median, range, and the size of 


\section{Obstetrics \& Gynecology Science}

Greg Marchand, et al. Systematic review of laparoscopy in interstitial pregnancy

a sample. BMC Med Res Methodol 2005;5:13.

21. Divry V, Hadj S, Bordes A, Genod A, Salle B. Case of progressive intrauterine twin pregnancy after surgical treatment of cornual pregnancy. Fertil Steril 2007;87:190.e1-3.

22. Dumesic DA, Damario MA, Session DR. Interstitial heterotopic pregnancy in a woman conceiving by in vitro fertilization after bilateral salpingectomy. Mayo Clin Proc 2001;76:90-2.

23. Garretto $D$, Lee $L N$, Budorick NE, Figueroa R. Interstitial twin pregnancy: a unique case presentation. J Clin Ultrasound 2015;43:447-50.

24. Garzon S, Laganà AS, Pomini P, Raffaelli R, Ghezzi F, Franchi M. Laparoscopic reversible occlusion of uterine arteries and cornuostomy for advanced interstitial pregnancy. Minim Invasive Ther Allied Technol 2019;28:359-62.

25. Gezer A, Mutlu H. Laparoscopic management of cornual pregnancy without sutures. Arch Gynecol Obstet 2004;270:194-6.

26. Grimbizis GF, Tsalikis T, Mikos T, Zepiridis L, Athanasiadis A, Tarlatzis BC, et al. Case report: laparoscopic treatment of a ruptured interstitial pregnancy. Reprod Biomed Online 2004;9:447-51.

27. Grobman WA, Milad MP. Conservative laparoscopic management of a large cornual ectopic pregnancy. Hum Reprod 1998;13:2002-4.

28. Kalchman GG, Meltzer RM. Interstitial pregnancy following homolateral salpingectomy. Report of 2 cases and a review of the literature. Am J Obstet Gynecol 1966;96:1139-43.

29. Kasum M, Grizelj V, Simunic V. Combined interstitial and intrauterine pregnancies after in-vitro fertilization and embryo transfer. Hum Reprod 1998;13:1547-9.

30. Ko ML, Jeng CJ, Chou CS, She BC, Chen SC, Tzeng CR. Laparoscopic electrodessication of an interstitial pregnancy. Fertil Steril 2007;88:705.e19-20.

31. Koukoura O, Dragoumis G, Gorila G, Gkorezi-Ntavela I, Dafopoulos K, Pistofidis G. Spontaneous intraoperative rupture of a large interstitial pregnancy: laparoscopic management. Case Rep Obstet Gynecol 2020 Apr 14 [Epub]. https://doi.org/10.1155/2020/5626783.

32. Kumakiri J, Takeuchi H, Kitade M, Kikuchi I, Shimanuki $H$, Kubo $M$, et al. Interstitial pregnancy with huge adenomyosis uteri managed laparoscopically by using pre-operative and intra-operative imaging: case report. BJOG 2005;112:1578-80.

33. Lai YJ, Lin CH, Hou WC, Hwang KS, Yu MH, Su HY. Pregnancy in a noncommunicating rudimentary horn of a unicornuate uterus: prerupture diagnosis and management. Taiwan J Obstet Gynecol 2016;55:604-6.

34. Lam PM, Lok IH, Yip SK. Two cases of heterotopic cornual pregnancy with initially missed diagnosis. Aust N Z J Obstet Gynaecol 2004;44:256-9.

35. Lee ES, Hahn HS, Park BJ, Ro DY, Kim JH, Kim YW. Single-port laparoscopic cornual resection for a spontaneous cornual ectopic pregnancy following ipsilateral salpingectomy. Fertil Steril 2011;96:e106-10.

36. Lialios GA, Kallitsaris A, Kabisios T, Messinis IE. Ruptured heterotopic interstitial pregnancy: rare case of acute abdomen in a Jehovah's Witness patient. Fertil Steril 2008;90:1200.e15-7.

37. Liao CY, Ding DC. Repair of uterine rupture in twin gestation after laparoscopic cornual resection. J Minim Invasive Gynecol 2009;16:493-5.

38. Maher PJ, Grimwade JC. Cornual pregnancy--diagnosis before rupture a report of 2 cases. Aust N Z J Obstet Gynaecol 1982;22:172-4.

39. Marfori CQ, Kotzen M. Angular vs. interstitial pregnancy: a case report highlighting diagnostic nuances with stark management differences. Case Rep Womens Health 2018;19:e00068.

40. Morita Y, Tsutsumi O, Momoeda M, Taketani Y. Cornual pregnancy successfully treated laparoscopically with fibrin glue hemostasis. Obstet Gynecol 1997;90 (4 Pt 2):685-7.

41. Nabeshima $H$, Nishimoto $M$, Utsunomiya $H$, Arai $M$, Ugajin T, Terada $Y$, et al. Total laparoscopic conservative surgery for an intramural ectopic pregnancy. Diagn Ther Endosc 2010 Oct 11 [Epub]. https://doi. org/10.1155/2010/504062.

42. Oelsner G, Admon D, Shalev E, Shalev Y, Kukia E, Mashiach S. A new approach for the treatment of interstitial pregnancy. Fertil Steril 1993;59:924-5.

43. Oral S, Akpak YK, Karaca N, Babacan A, Savan K. Cornual heterotopic pregnancy after bilateral salpingectomy and uterine septum resection resulting in term delivery of a healthy infant. Case Rep Obstet Gynecol 2014 Nov 5 [Epub]. https://doi. org/10.1155/2014/157030. 


\title{
Obstetrics \& Gynecology Science
}

\author{
Vol. 64, No. 2, 2021
}

44. Osuga $Y$, Tsutsumi O, Fujiwara T, Kugu K, Fujimoto A, Taketani Y. Usefulness of long-jaw forceps in laparoscopic cornual resection of interstitial pregnancies. J Am Assoc Gynecol Laparosc 2001;8:429-32.

45. Pasic RP, Hammons G, Gardner JS, Hainer M. Laparoscopic treatment of cornual heterotopic pregnancy. J Am Assoc Gynecol Laparosc 2002;9:372-5.

46. Pedroso C, Lermann R, Amaral N, Condeço P. Interstitial pregnancy rupture at 15 weeks of pregnancy. BMJ Case Rep 2014 Aug 25 [Epub]. https://doi. org/10.1136/bcr-2014-203979.

47. Pluchino N, Ninni F, Angioni S, Carmignani A, Genazzani AR, Cela V. Spontaneous cornual pregnancy after homolateral salpingectomy for an earlier tubal pregnancy: a case report and literature review. J Minim Invasive Gynecol 2009;16:208-11.

48. Poujade O, Ducarme G, Luton D. Cornual heterotopic pregnancy: a case report. J Med Case Rep 2009 Jun 23 [Epub]. https://doi.org/10.4076/1752-1947-3-7233.

49. Tsuchiya A, Komatsu Y, Matsuyama R, Tsuchiya $H$, Takemura $Y$, Nishii O. Intraoperative and postoperative clinical evaluation of the hysteroscopic morcellator system for endometrial polypectomy: a prospective, randomized, single-blind, parallel group comparison study. Gynecol Minim Invasive Ther 2018;7:16.

50. Ron-el R, Langer R, Herman A, Caspi E, Bukovsky Y. Term delivery following mid-trimester ruptured cornual pregnancy with combined intrauterine pregnancy. Case report. Br J Obstet Gynaecol 1988;95:619-20.

51. Sagiv R, Golan A, Arbel-Alon S, Glezerman M. Three conservative approaches to treatment of interstitial pregnancy. J Am Assoc Gynecol Laparosc 2001;8:154-8.

52. Rheinboldt $M$, Ibrahim S. Atypical presentation of a large interstitial pregnancy. Emerg Radiol 2013;20:251-4.

53. Raheem M, Afifi Y. Laparoscopic selective ipsilateral uterine artery ligation for the management of a cornual ectopic pregnancy. J Minim Invasive Gynecol 2008;15:260-1.

54. Quinlan D, Newcombe M. Cornual ectopic pregnancy. J Obstet Gynaecol Can 2007;29:537-8.

55. Ross R, Lindheim SR, Olive DL, Pritts EA. Cornual gestation: a systematic literature review and two case reports of a novel treatment regimen. J Minim Invasive Gynecol 2006;13:74-8.

56. Sahoo S, Jose J, Shah N, Opemuyi I. Recurrent cornual ectopic pregnancies. Gynecol Surg 2009;6:389-91.

57. Sant CLH, Andersen PE. Misdiagnosed uterine rupture of an advanced cornual pregnancy. Case Rep Radiol 2012 Apr 3 [Epub]. https://doi. org/10.1155/2012/289103.

58. Sarmini RO, Tate D. Ruptured left cornual gestation in an unstable patient. J Minim Invasive Gynecol 2005; 12:383.

59. Sherer DM, Scibetta JJ, Sanko SR. Heterotopic quadruplet gestation with laparoscopic resection of ruptured interstitial pregnancy and subsequent successful outcome of triplets. Am J Obstet Gynecol 1995;172:216-7.

60. Takeda A, Koyama K, Imoto S, Mori M, Sakai K, Nakamura $\mathrm{H}$. Successful management of interstitial pregnancy with fetal cardiac activity by laparoscopicassisted cornual resection with preoperative transcatheter uterine artery embolization. Arch Gynecol Obstet 2009;280:305-8.

61. Tinelli A, Malvasi A, Pellegrino $M$, Pontrelli $G$, Martulli $B$, Tsin DA. Laparoscopical management of cornual pregnancies: a report of three cases. Eur J Obstet Gynecol Reprod Biol 2010;151:199-202.

62. Ugwumadu AHN, Hamid R, Ross LD. Live infant salvaged from a ruptured cornual (interstitial) pregnancy at 33-weeks gestation. Int J Gynecol Obstet 1997;58:247-9.

63. Vicino M, Loverro G, Resta L, Bettocchi S, Vimercati A, Selvaggi L. Laparoscopic cornual excision in a viable large interstitial pregnancy without blood flow detected by color Doppler ultrasonography. Fertil Steril 2000;74:407-9.

64. Vilos GA. Laparoscopic resection of a heterotopic cornual pregnancy followed by term vaginal delivery. J Am Assoc Gynecol Laparosc 1995;2:471-3.

65. Vilos GA. Laparoscopic ligation and resection of two ipsilateral interstitial pregnancies in the same patient. J Am Assoc Gynecol Laparosc 2001;8:299-302.

66. Chopra S, Keepanasseril A, Rohilla M, Bagga R, Kalra J, Jain V. Obstetric morbidity and the diagnostic dilemma in pregnancy in rudimentary horn: retrospective analysis. Arch Gynecol Obstet 2009;280:907-10.

67. Weissman A, Fishman A. Uterine rupture following conservative surgery for interstitial pregnancy. Eur J Obstet Gynecol Reprod Biol 1992;44:237-9.

68 . Wood C, Hurley V. Ultrasound diagnosis and laparo- 


\section{Obstetrics \& Gynecology Science}

Greg Marchand, et al. Systematic review of laparoscopy in interstitial pregnancy

scopic excision of an interstitial ectopic pregnancy. Aust N Z J Obstet Gynaecol 1992;32:371-2.

69. Woodland MB, DePasquale SE, Molinari JA, Sagullo CC. Laparoscopic approach to interstitial pregnancy. J Am Assoc Gynecol Laparosc 1996;3:439-41.

70. Yalçın Y, Tatar B, Erdemoğlu E, Akkurt MÖ, Yavuz A, Erdemoğlu E. Laparoscopic systemic devascularization of uterine cornu for cornual resection in interstitial pregnancy. Turk J Obstet Gynecol 2015;12:182-4.

71. Yang $H$, Song T. Temporary simultaneous 2 arterial occlusions during laparoscopic management for cornual ectopic pregnancy. J Minim Invasive Gynecol 2018;25:961-2.

72. Zhang $X$, Liu $X$, Fan H. Interstitial pregnancy and transcervical curettage. Obstet Gynecol 2004;104(5 Pt 2):1193-5.

73. Zhang K, Yuan P. Laparoscopy-assisted vaginal cornual resection for the treatment of large interstitial pregnancy. J Laparoendosc Adv Surg Tech A 2013;23:783-6.

74. Api M, Api O. Laparoscopic cornuotomy in the management of an advanced interstitial ectopic pregnancy: a case report. Gynecol Endocrinol 2010;26:208-2.

75. Attia M, Karuppaswamy J, Griffith H. Management of interstitial (cornual) pregnancy at 17 weeks' gestation: conservation of a ruptured uterus. J Obstet Gynaecol 2005;25:722-3.

76. Bremner T, Cela V, Luciano AA. Surgical management of interstitial pregnancy. J Am Assoc Gynecol Laparosc 2000;7:387-9.

77. Cai Z, Wang F, Cao H, Xia Q. Transcervical suction of interstitial pregnancy under laparoscopic and hysteroscopic guidance. J Minim Invasive Gynecol 2009;16:761-4.

78. Casadio P, Formelli G, Spagnolo E, De Angelis D, Marra E, Armillotta $F$, et al. Laparoscopic treatment of interstitial twin pregnancy. Fertil Steril 2009;92:390.e13-7.

79. Chachan S, Waters N, Kent A. Laparoscopic management of cornual heterotopic pregnancy with the use of Harmonic ACE $®$-a case report. Gynecol Surg 2011;8:243-6.

80. Chauhan M, Chaudhary P, Dahiya P, Sangwan K, Sen J. Molar cornual ectopic pregnancy. Acta Obstet Gynecol Scand 2006;85:625-6.

81. Cheng Z, Xu L, Zhu Y, Dai H, Qu X, Gong J. Laparoscopic uterine vessels occlusion for the treatment of interstitial pregnancy. J Laparoendosc Adv Surg Tech A 2009;19:509-12.

82. Chin HY, Chen FP, Wang CJ, Shui LT, Liu YH, Soong YK. Heterotopic pregnancy after in vitro fertilizationembryo transfer. Int J Gynecol Obstet 2004;86:411-6.

83. Corić M, Barisić D, Strelec M. Laparoscopic approach to interstitial pregnancy. Arch Gynecol Obstet 2004;270:287-9.

84. Dendas W, Schobbens JC, Mestdagh G, Meylaerts L, Verswijvel G, Van Holsbeke C. Management and outcome of heterotopic interstitial pregnancy: case report and review of literature. Ultrasound 2017;25:134-42.

85. Di Tizio L, Spina MR, Gustapane S, D'Antonio F, Liberati M. Interstitial pregnancy: from medical to surgical approach-report of three cases. Case Rep Obstet Gynecol 2018 Oct 15 [Epub]. https://doi. org/10.1155/2018/2815871.

86. Cucinella G, Rotolo S, Calagna G, Granese R, Agrusa A, Perino A. Laparoscopic management of interstitial pregnancy: the "purse-string" technique. Acta Obstet Gynecol Scand 2012;91:996-9.

87. Zuo X, Shen A, Chen M. Successful management of unruptured interstitial pregnancy in 17 consecutive cases by using laparoscopic surgery. Aust N Z J Obstet Gynaecol 2012;52:387-90.

88. Gao B, Cheng C, Pan Q, Johnson G, Qin X, Xu D. Laparoscopic strategy for heterotopic interstitial pregnancy following assisted reproductive techniques. JSLS 2019 Apr-Jun [Epub]. https://doi.org/10.4293/ JSLS.2018.00109.

89. MacRae R, Olowu O, Rizzuto MI, Odejinmi F. Diagnosis and laparoscopic management of 11 consecutive cases of cornual ectopic pregnancy. Arch Gynecol Obstet 2009;280:59-64.

90. Tulandi T, Al-Jaroudi D. Interstitial pregnancy: results generated from the society of reproductive surgeons registry. Obstet Gynecol 2004;103:47-50.

91. Watanabe T, Watanabe Z, Watanabe T, Fujimoto K, Sasaki E. Laparoscopic cornuotomy for interstitial pregnancy and postoperative course. J Obstet Gynaecol Res 2014;40:1983-8.

92. Uludag SZ, Kutuk MS, Dolanbay M, Ozgun MT, Eliyeva $\mathrm{G}$, Altun O. Conservative management of interstitial pregnancies: experience of a single centre. J Obstet Gynaecol 2018;38:848-53. 


\title{
Obstetrics \& Gynecology Science
}

\author{
Vol. 64, No. 2, 2021
}

93. Sagiv R, Debby A, Keidar R, Kerner R, Golan A. Interstitial pregnancy management and subsequent pregnancy outcome. Acta Obstet Gynecol Scand 2013;92:1327-30.

94. Chen PL, Lin HH, Hsiao SM. Predictors of subsequent pregnancy in women who underwent laparoscopic cornuostomy or laparoscopic wedge resection for interstitial pregnancy. J Chin Med Assoc 2019;82:13842.

95. Xu W, Lin X, Huang D, Zhang S. Laparoscopic treatment of cornual heterotopic pregnancy: a retrospective cohort study. Int J Surg 2018;53:98-102.

96. Choi YS, Eun DS, Choi J, Shin KS, Choi JH, Park HD. Laparoscopic cornuotomy using a temporary tourniquet suture and diluted vasopressin injection in interstitial pregnancy. Fertil Steril 2009;91:1933-7.

97. Olagundoye V, Adeghe J, Guirguis M, Cox C, Murphy D. Laparoscopic surgical management of ectopic pregnancy: a district general hospital experience. J Obstet Gynaecol 2000;20:620-3.

98. Ng S, Hamontri S, Chua I, Chern B, Siow A. Laparoscopic management of 53 cases of cornual ectopic pregnancy. Fertil Steril 2009;92:448-52.

99. Wang YL, Weng SS, Huang WC, Su TH. Laparoscopic management of ectopic pregnancies in unusual locations. Taiwan J Obstet Gynecol 2014;53:466-70.

100. Grant A, Murji A, Atri M. Can the presence of a surrounding endometrium differentiate eccentrically located intrauterine pregnancy from interstitial ectopic pregnancy? J Obstet Gynaecol Can 2017;39:627-34.

101. Kim MJ, Jung YW, Cha JH, Seok HH, Han JE, Seong SJ, et al. Successful management of heterotopic cornual pregnancy with laparoscopic cornual resection. Eur J Obstet Gynecol Reprod Biol 2016;203:199-203.

102. Mencaglia L, Tiso E, Tantini C, Bianchi R. Risks of virus transmission during diagnostic hysteroscopy. J Am Assoc Gynecol Laparosc 1996;3(4, Supplement):S30.

103. Moon HS, Choi YJ, Park YH, Kim SG. New simple endoscopic operations for interstitial pregnancies. Am J Obstet Gynecol 2000;182:114-21.

104. Moon HS, Kim SG, Park GS, Choi JK, Koo JS, Joo BS. Efficacy of bleeding control using a large amount of highly diluted vasopressin in laparoscopic treatment for interstitial pregnancy. Am J Obstet Gynecol 2010;203:30.e1-6.
105. Nirgianakis K, Papadia A, Grandi G, McKinnon B, Bolla $D$, Mueller MD. Laparoscopic management of ectopic pregnancies: a comparison between interstitial and "more distal" tubal pregnancies. Arch Gynecol Obstet 2017;295:95-101.

106. Ghazali WAHW, Abidin NHZ, Muda AM, Hamid HA. Comparative study on surgical outcomes between laparoscopic and open cornuotomy in urban tertiary center of Malaysia. Gynecol Minim invasive Ther 2018;7:22-6.

107. Lazard A, Poizac S, Courbiere B, Cravello L, Gamerre M, Agostini A. Cornual resection for interstitial pregnancy by laparoendoscopic single-site surgery. Fertil Steril 2011;95:2432.e5-8.

108. Huang MC, Su TH, Lee MY. Laparoscopic management of interstitial pregnancy. Int J Gynaecol Obstet 2005;88:51-2.

109. Yoong W, Neophytou C, de Silva L, Adeyemo A, Lodhi W. Novel laparoscopic cornual resection of interstitial pregnancy using the Endo GIATM Universal Stapler (Medtronic): a series of 12 cases. Aust N Z J Obstet Gynaecol 2020;60:130-4.

110. Kahramanoglu I, Mammadov Z, Turan H, Urer A, Tuten A. Management options for interstitial ectopic pregnancies: a case series. Pak J Med Sci 2017;33:476-82.

111. Said TH. Laparoscopic management of interstitial ectopic using simple and safe technique: case series and review of literature. J Obstet Gynaecol India 2016;66(Suppl 1):482-7.

112. Faioli R, Berretta R, Dall'Asta A, Di Serio M, Galli L, Monica $M$, et al. Endoloop technique for laparoscopic cornuectomy: a safe and effective approach for the treatment of interstitial pregnancy. J Obstet Gynaecol Res 2016;42:1034-7.

113. Hwang JH, Lee JK, Lee NW, Lee KW. Open cornual resection versus laparoscopic cornual resection in patients with interstitial ectopic pregnancies. Eur J Obstet Gynecol Reprod Biol 2011;156:78-82.

114. Kim MK, Kim JJ, Choi JS, Eom JM, Lee JH. Prospective comparison of single port versus conventional laparoscopic surgery for ectopic pregnancy. J Obstet Gynaecol Res 2015;41:590-5.

115. Cucinella G, Calagna G, Rotolo S, Granese R, Saitta S, Tonni $G$, et al. Interstitial pregnancy: a 'road map' of surgical treatment based on a systematic review of the 


\section{Obstetrics \& Gynecology Science}

Greg Marchand, et al. Systematic review of laparoscopy in interstitial pregnancy

literature. Gynecol Obstet Invest 2014;78:141-9.

116. Gao M, Gao Q, Jia J, Bao L, Zhang P, Tian JH, et al. Laparoscopy versus laparotomy for ectopic pregnancy: a systematic review. Chinese Journal of EvidenceBased Medicine 2009;9:994-1000.

117. Ehrenberg-Buchner S, Sandadi S, Moawad NS, Pinker- ton JS, Hurd WW. Ectopic pregnancy: role of laparoscopic treatment. Clin Obstet Gynecol 2009;52:372-9.

118. Rempen A, Dietl J. Complication rates after surgical treatment of ectopic pregnancy. Hum Reprod 1999;14:1401-3. 